\title{
The incidence of symptomatic deep vein thrombosis and pulmonary embolism in the Indian population, following TKA patients, with postoperative prophylaxis
}

\author{
Sandeep Krishna Avulapati ${ }^{1}$, Senthil Kumar Mahalingam ${ }^{2, *}$, Jagdeesh Gudaru ${ }^{3}$, Karthik Gudaru ${ }^{4}$ \\ ${ }_{1,2,4}$ Assistant Professor, ${ }^{3}$ Director, Professor and HOD, Dept. of Orthopedics, BIRRD (T) Hospital, Sri Padmavathi Medical \\ College for Women, SVIMS University, Andhra Pradesh, India
}

*Corresponding Author: Senthil Kumar Mahalingam

Email: drsenthil72@gmail.com

\begin{abstract}
Introduction: Deep Vein Thrombosis (DVT) and Pulmonary Embolism (PE) are the life-threatening complications which can occur in patients undergoing elective Total Knee Arthroplasties (TKA). Incidence in western population appears high in published Literature. While the Indian population it appears to be variable. The difference might be due to different Genetic makeup, social lifestyles of both populations. ${ }^{1}$ The study intends to find an incidence of symptomatic DVT and PE in postoperative TKA patients on the prophylactic drug regimen.

Aim: To study the incidence of symptomatic DVT and PE, in post-TKA patients in Indian population.

Materials and Methods: Retrospective Study conducted from $1^{\text {st }}$ may, 2017 to 1st may 2018 in BIRRD (T) Hospital. All patients who underwent TKA are included, after following inclusion and exclusion criteria. These patients were evaluated for symptoms during the hospital stay and after discharge for 3 Months.

Result: Total number of patients who had TKA were 2292(N=2292). The symptomatic DVT was found in 6(n=6) patients. All 3 patients were managed with a therapeutic regimen. Four patients developed $\mathrm{PE}(\mathrm{p}=4)$ during the study period, three patients developed pulmonary embolism in first 24 hours postoperatively and expired in spite of ICU management. One patient expired on the $4^{\text {th }}$-week postoperatively, with PE at home. Two patients presented with symptoms on $2^{\text {nd }}$ and $4^{\text {th }}$ postoperative days, one underwent fasciotomy on $4^{\text {th }}$ postoperative day. All other patients were on a prophylactic regimen.
\end{abstract}

Conclusion: Our results suggest incidence of DVT and PE are low in the Indian population with a prophylactic regimen.

Keywords: Deep vein thrombosis, Pulmonary embolism, Prophylaxis, Total knee arthroplasties, Venous thromboembolism.

\section{Introduction}

Deep vein thrombosis and Pulmonary embolism are the two life-threatening complications from elective joint replacements mainly Knee arthroplasties in the Western world. Nearly 300000-600000 patients are hospitalized every year in the United States due to DVT and pulmonary embolism with mortality at about 50000 patients. $^{2}$ incidence of post operative DVT for Knee arthroplasties is $0.22 \%$ which is quite higher than Hip arthroplasties $(0.15 \%)$ and fracture hip surgeries $(0.16 \%)^{3}$

The incidence of DVT and PE appears to be low in our study because of genetic makeup and lifestyle differences. Indian population is mostly rural $(2 / 3$ of the population live in villages, with the farming as main occupation, belong to poor socio-economic status. They do have a physically demanding lifestyle and present at a late age with Osteoarthritis, of knee. ${ }^{6}$ patients who undergo TKA in our institute are mostly from rural background. Presence of risk factors of DVT and PE is less in rural population than urban population like sedentary lifestyle $3.3 \%$ to $13.7 \%$, smoking $13.4 \%$ to $19.2 \%$, obesity $5.4 \%$ to $12.7 \%$, diabetes $10 \%$ to $14.4 \%$.

The incidence of Osteoarthritis in US population ranges from 55-64years (with median age 55years), ${ }^{8}$ while in the India it is 45-88 years (with median age 64.4years). ${ }^{1,9}$ Based on the differences between the two populations, we do expect the incidence of DVT and Pulmonary embolism are high because of the high mean age, in Indian scenario. Moreover, drugs used as a prophylactic measure are costly and their use is limited in Indian population due to lack of adequate health facilities in a rural set-up, poor socio-economic conditions, illiteracy here. Results of various studies conducted in India shows variable incidence because of small sample sizes. Hence we have performed a study with a large sample size to ascertain the more accurate incidence of DVT and PE in the Indian population.

Clinical Diagnosis: Patients were diagnosed DVT clinically based on symptoms like pain (Homan's sign: Calf pain while dorsiflexing the foot), swelling, tenderness, warmth, leg cramps and skin discoloration. Patients with PE are diagnosed clinically by Tachypnea, Tachycardia, dyspnoea, chest discomfort, and acute onset of pleuritic chest pain, cough and fever. ${ }^{5}$ DVT cases present the second day to $6^{\text {th }}$ postoperative weeks, where PE presents in first post operative week. ${ }^{5}$

Treatment: In India use of anticoagulant medication is the mainstay in the treatment of VTE where 5 days of subcutaneous anticoagulation therapy followed by longterm (3 months) of Vitamin K antagonist orally. ${ }^{5}$

\section{Materials and Methods}

This was a retrospective study done. All TKR patients done in BIRRD (T) hospital from $1^{\text {st }}$ May 2017 to $1^{\text {st }}$ May 2018 were included in the study. 2302 patients were evaluated for the DVT symptoms postoperatively during the course of the hospital stay up to 7 days, post-discharge for 3 months. 


\section{Inclusion Criteria:}

1. All patients underwent TKA in the BIRRD (T) Hospital.

2. Patients with age $>50$ years.

3. Complex primary TKA surgeries.

\section{Exclusion Criteria:}

1. History of venous thromboembolism.

2. Varicose veins.

3. Revision TKA surgeries.

Patients on admission were evaluated for Hypertension, diabetes, smoking, malignancy. Patients with a history of thromboembolism were excluded from the study. Patients were evaluated in Cardiology, Anaesthesiology prior to surgery. Patients were taken up for surgery after the fitness from respective departments.

We follow the following protocol for Chemoprophylaxis for DVT, in BIRRD (T) Hospital for the TKA patients.

1. Intra-operatively we are not using mechanical devices such as calf pumps, compression stockings during the procedure due to cost implication and low incidence

2. Up to 24 hours of surgery no anticoagulation therapy started due to epidural catheter for pain relief and postoperative mobilization.

3. After 24 hours of surgery, we start Low Molecular Weight Heparin for 5 days or to the day of discharge (whichever is the last) after removal of epidural catheter (6 hours after removal of epidural catheter). Due to practical reasons, we don't recommend continuation of LMV Heparin at home.

4. From the day of discharge, we switch over to oral anticoagulant tab Rivaroxaban $15 \mathrm{mg}$ twice daily for 14days from the $8^{\text {th }}$ postoperative day.

5. Ankle and calf exercises started soon after surgery.

6. Quadriceps and hamstrings exercises, ROM of ankle and knee movements, partial wt bearing walking exercises on the first postoperative day.

7. Full wt bearing walk with walker support and mobilization of patients from the $2^{\text {nd }}$ postoperative day.
Patients were evaluated for DVT symptoms such as Homan's sign, ankle and leg swelling, tenderness, warmth, leg cramps and discoloration of skin. Fever and respiratory symptoms (dyspnoea, chest discomfort and pleuritic chest pain) for PE. Patients were followed up at 6 weeks, 3 months and 6 months thereafter. They were evaluated for symptoms during these visits.

\section{Observations and Results}

In our study, we have included at about 2302 patients in our study. There were 1129 male and 1164 female patients. We had a difference in opinion regarding fitness for surgery from Anesthesia and Cardiology departments and could go forward with surgery in 9 patients (one male and eight female), because of high risk for surgery for elective TKA. The average age of patients in our study is 62 years (male $=64$ years and female $=60$ years). Patients undergoing right TKR are 1171 and left TKA are 1121. There is no significant difference between Right and Left TKA in male and female population.

In our institute, we don't take up bilateral TKA, in order to prevent postoperative complications which double with single surgeries. We do see that the gap between two TKA is at about 6 months minimum. The incidence of DVT increases in Bilateral TKA (36.9\%) than that of Unilateral TKA $(25.6 \%)$ as shown in the study conducted by Richard F O'Reilly (2005) et al. Of these 2293 surgeries, 24 patients had complex TKA with use of Stem for stability, augments which are either bone or metal wedges for covering defective sites in femur and tibia. Average operating time for TKA is 105 minutes (90-120 minutes), with tourniquet time at about 75 minutes (60-90 minutes). Post surgery we do check up lower limb pulses, $\mathrm{SpO}_{2}$ as a part of institute protocol. We use Intrarticular drains during the procedure were estimated blood loss is at about $200 \mathrm{ml}$ during surgery and less than $200 \mathrm{ml}$ post surgery. Drains are removed after 24 hours of the procedure.

Table 1: No of patients (male and female), average age, no. of right and left TKA done

\begin{tabular}{|l|c|c|c|c|c|}
\hline \multicolumn{1}{|c|}{ Sex } & No of Patients & Average age & RT TKA & LT TKA & Total \\
\hline Male & $1130(1)^{*}$ & 64 years & $550(3)^{* *}$ & $579(11)^{* *}$ & $1129(14)^{* *}$ \\
\hline Female & $1172(8)^{*}$ & 60 years & $621(1)^{* *}$ & $543(9)^{* *}$ & $1164(10)^{* *}$ \\
\hline Total & $2302(9)^{*}$ & 62 years & $1171(4)^{* *}$ & $1121(20)^{* *}$ & $2293(24)^{* *}$ \\
\hline
\end{tabular}

$(*)$ value in brackets show patients not posted for surgery

$(* *)$ value in brackets shows complicated TKA

In our study, the incidence of symptomatic DVT is $0.3 \%$ (6 out of 2293 patients) and PE $0.2 \%$ (4 out of 2293 patients). Mortality incidence is about $0.2 \%$ (4 deaths over 2293 patients). Morbidity is negligent $0.04 \%(1 / 2293)$. The reported number of DVT cases in our study is 6 with $4 \mathrm{PE}$ patients. Number deaths reported in our study are 4 . Sudden death was reported in 3 patients (one male and two female patients) on 612 hours after surgery. One female patient expired 4 weeks after surgery due to Pulmonary Embolism at her home town. She was on DVT Prophylaxis. One male patient was diagnosed DVT on the $4^{\text {th }}$ Postoperative day. Another Male patient was diagnosed on the $2^{\text {nd }}$ postoperative day. He underwent fasciotomy on the $4^{\text {th }}$ postoperative day under plastic surgery department, due 
to compartment syndrome. He later underwent split skin thickness grafting. Presently patient has recovered

and walking normally.

Table 2: Incidence of DVT, PE and morbidity and mortality in male, female and total number of patients in our study

\begin{tabular}{|l|c|c|c|c|}
\hline \multicolumn{1}{|c|}{ Sex } & $\begin{array}{c}\text { Deep Vein } \\
\text { Thrombosis }\end{array}$ & $\begin{array}{c}\text { Pulmonary } \\
\text { Embolism }\end{array}$ & Morbidity & Mortality \\
\hline Male & $3(0.27 \%)$ & $1(0.09 \%)$ & $1(0.09 \%)$ & $1(0.09 \%)$ \\
\hline Female & $3(0.26 \%)$ & $3(0.26 \%)$ & $0(0 \%)$ & $3(0.26 \%)$ \\
\hline Total & $6(0.3 \%)$ & $4(0.2 \%)$ & $1(0.05 \%)$ & $4(0.2 \%)$ \\
\hline
\end{tabular}

(Values in brackets shows incidence in that group)

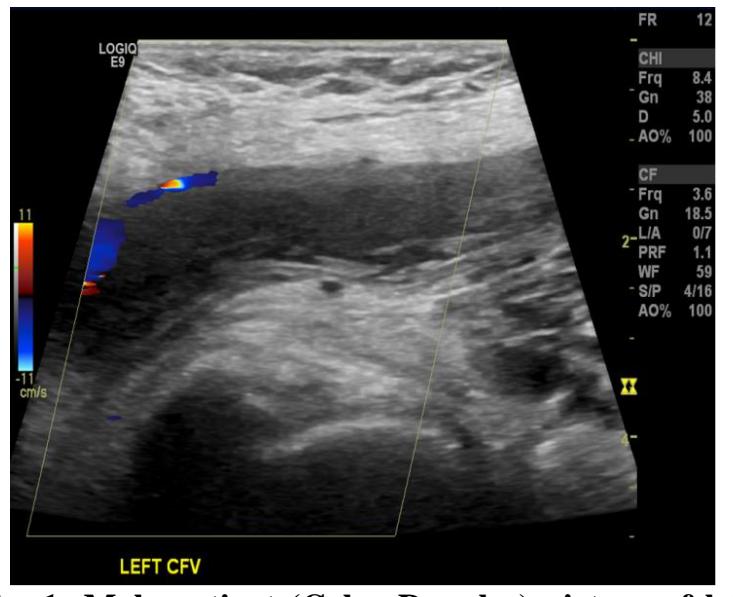

Fig. 1: Male patient (Color Doppler) picture of left common saphenous vein with no flow observed

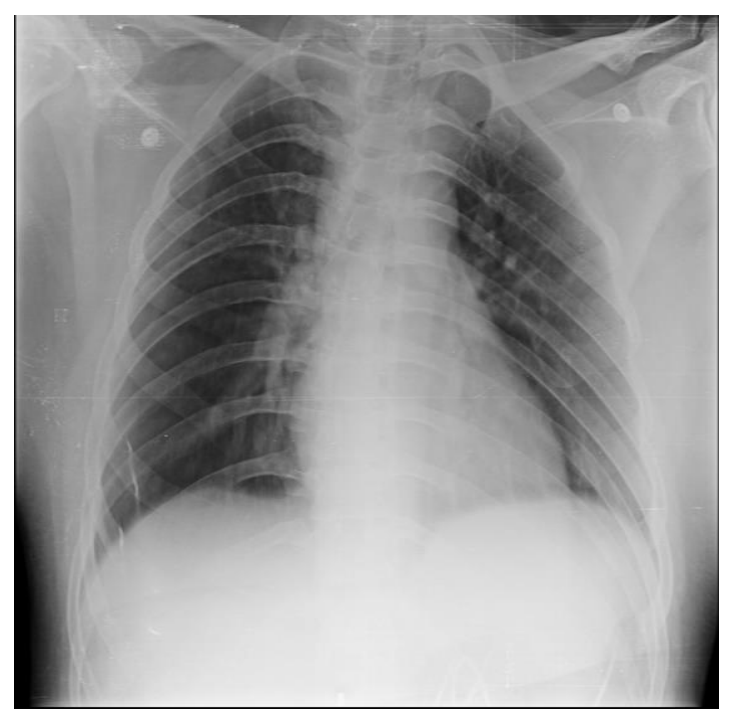

Fig. 2: Chest X-Ray of male patient taken immediately after the complaint of Breathless showing Oligaemia (PE) (decreased vascular marking) over right lower lobe of lung

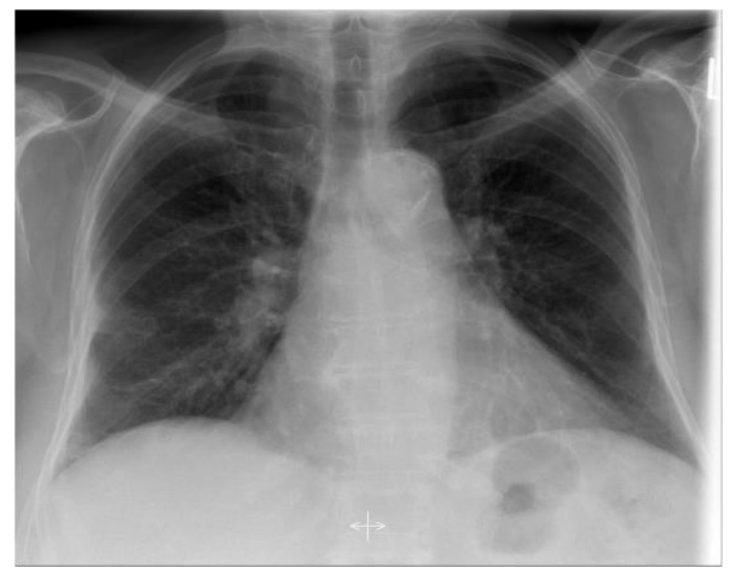

Fig. 3: Male patient which shows wedge shaped infarct area over the same zone of lung (progressed lesion)

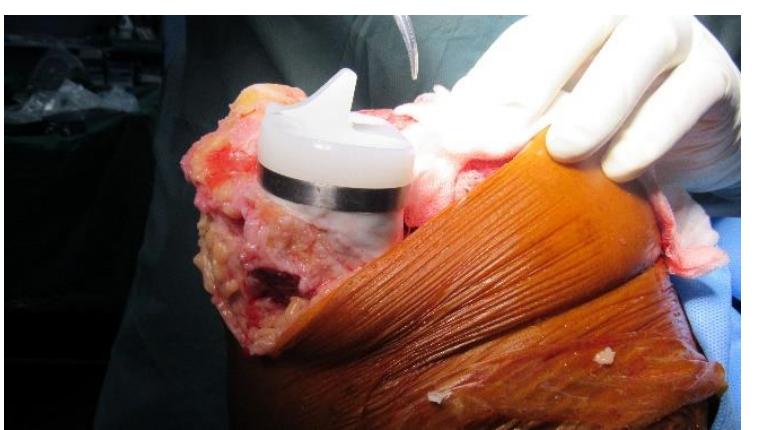

Fig. 4: Fixation of tibial implant during the procedure

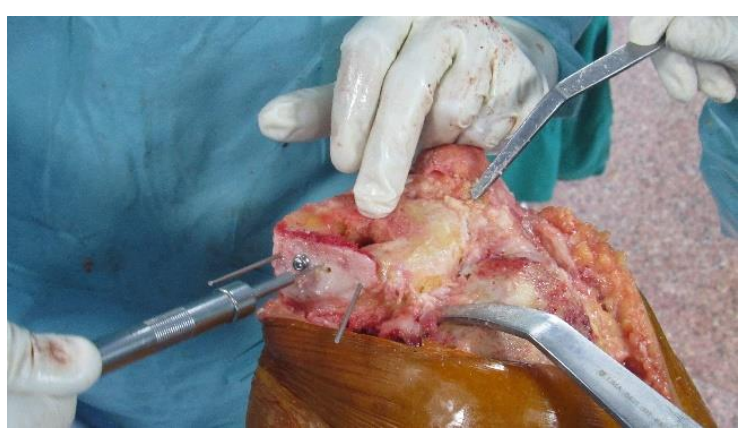

Fig. 5: Intra operative defective repair with grafting on the medial side of tibia 


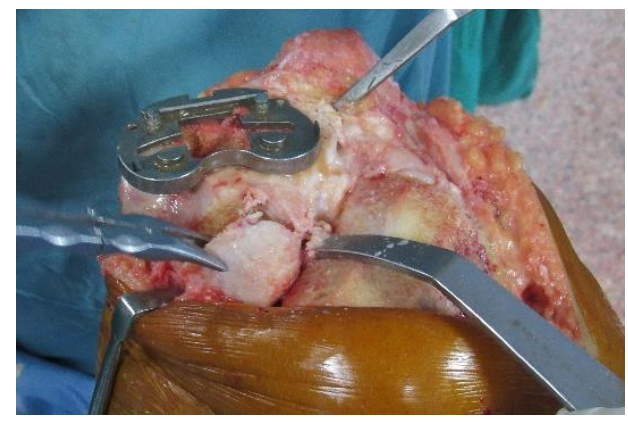

Fig. 6: Intra operative defective medial side of tibia and posterior big osteophyte which is being removal

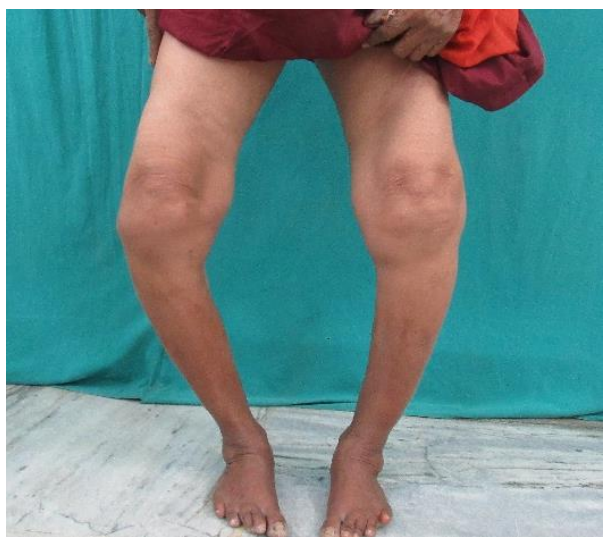

Fig. 7: Photograph of patient in standing position from the front planned for Right TKR

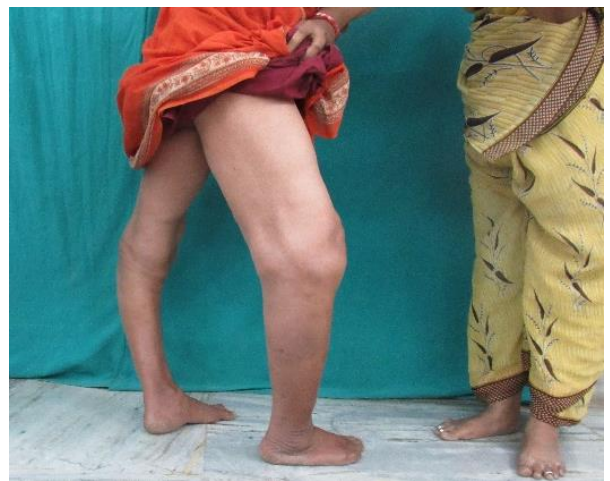

Fig. 8: Photograph of the patient in standing from the lateral side of patient planned Rt TKR

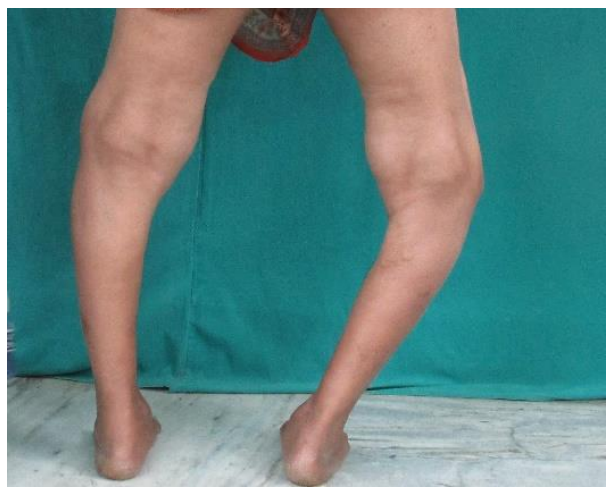

Fig. 9: Photograph of the patient standing from the back planned for Right TKR

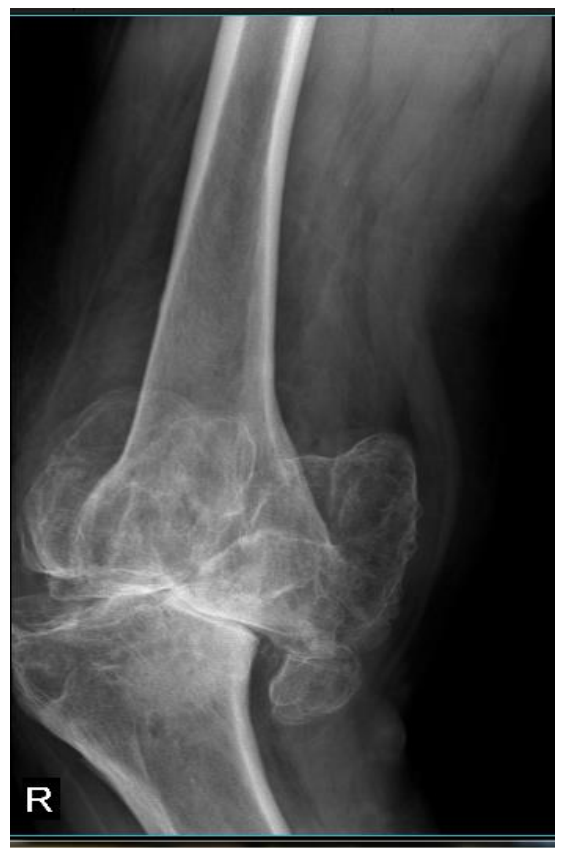

Fig. 10: X-ray of the patient AP view of Right knee

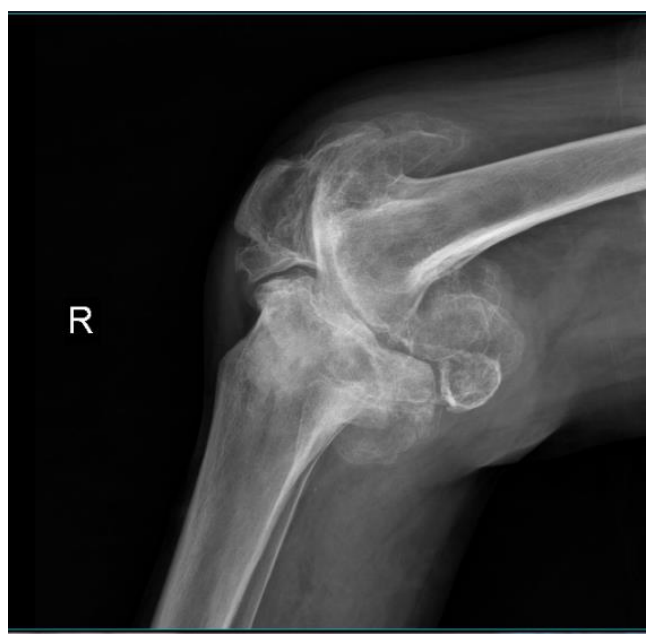

Fig. 11: X-ray of the patient lateral view Right side

\section{Discussion}

Here in our Institute, we do perform 12 TKA and 2-3 THA per day, 6 days a week, more than 3000 cases year. We in our Institute do have own customized thromboprophylaxis protocol which is not standardized. Our Institute is Charity Institute with TKA performed most of the cases. This is because we have patients who are from rural background, low socio-economic status, with minimal primary health care at the village, most of the population uneducated. Patients are given LMV Heparin injections 6 hours after epidural catheter removal, up to 5 days or to the day of discharge (whichever is the last). They will be discharged with oral Rivaroxaban $15 \mathrm{mg}$ twice daily for 14days. Patients were started oral, as per reasons mentioned above, and it is very easy for them to take medications orally than having subcutaneous heparin injections. Patients were 
examined for DVT and PE symptoms throughout stay in the hospital.

Our incidence of DVT is $0.3 \%$ is low when compared with other studies $3.96 \%$ (Nachiketan et al), ${ }^{4}$ $0 \%$ (V.Bagaria et.al, ${ }^{10}$ and V. Jain et al, ${ }^{1}$ ). Previously it was thought that incidence of DVT was high in Western population due to their Genetic makeup, lifestyle, and food habits when compared to Indian population. This is due to paucity of data and absence of clinical material in India. Present studies show variable incidence in the Indian population (i.e, incidence in patients with and without prophylaxis being $43 \%$ and $60 \%$ respectively). ${ }^{14}$ Shortcoming our study is that we may have missed large asymptomatic, diagnosed only USG, venography, Doppler and other investigations.

The incidence of symptomatic DVT to asymptomatic DVT is low as shown in the following studies. In the study conducted by Ye Sun et.al, incidence of symptomatic DVT is $3.7 \%$ (20 out 537 TKA) to asymptomatic DVT $11.2 \%$ (60/537). ${ }^{12}$ In study by D.J. Quinlan et al, study ratio of symptomatic to asymptomatic is $1 ; 21$ (i.e, is one out 21 asymptomatic DVT patients become symptomatic DVT) in TKA patients. ${ }^{13}$

In a study by Won. Y.S. et al, shows that out of 534 subjects (524 subjects on DVT chemoprophylaxis) who underwent TKA, DVT was reported in 9 patients, 8 DVT in the lower limb, and one had a pulmonary embolism. Here the ratio of symptomatic to asymptomatic DVT is $3(0.56 \%)$ : $5(0.94 \%) .{ }^{15}$

Data of 23,475 TKA patients (837 citation of 47 studies were included) conducted across from January 1st, 1996 to September 30th, 2011 (collected across Medline, Embase and Cochrane library), shows symptomatic VTE occurred in 288 patients (incidence $1.2 \%)$ and pulmonary embolism in 182 patients (incidence $0.78 \%$ ). All the patients in the study were on chemoprophylaxis. ${ }^{16}$

Incidence in our study $0.3 \%(6 / 2302)$ is comparable to other Asian studies like $0.22 \%$ (Seung Yeol Lee et $\mathrm{al}^{3}$ ), 3.7\% (Ye Sun et al), ${ }^{12}$ and $0.56 \%$ (Won. Y.S. et al,). ${ }^{15}$

Table 3: Incidence in various parts of India, Asia, world with their sample size, year of study

\begin{tabular}{|c|c|c|c|c|}
\hline Study & Incidence & Sample size & Year & Region \\
\hline \multicolumn{5}{|c|}{ Indian Studies } \\
\hline Nachiketan et al, ${ }^{4}$ & $3.96 \%$ & 101 & 2017 & India \\
\hline Tom Jose et al, ${ }^{18}$ & $0.91 \%$ & 100 & 2017 & India \\
\hline V.Bragara et $a{ }^{10}{ }^{10}$ & $0 \%$ & 29 & 2006 & India \\
\hline V. Jain et al, ${ }^{1}$ & $0 \%$ & 46 & 2004 & India \\
\hline \multicolumn{5}{|c|}{ Other Asian Studies } \\
\hline Seung Yeol et al, ${ }^{3}$ & $0.22 \%$ & 180611 & 2015 & Korea (Asia) \\
\hline Piovella et al, ${ }^{11}$ & $58.1 \%$ & 136 & 2005 & Multi ethnic study (Asia) \\
\hline D. J. Quinlan et al, ${ }^{13}$ & $4.5 \%$ & 22(asymptomatic) & 2007 & Asia \\
\hline Ye Sun, M.D et al, ${ }^{12}$ & $3.7 \%$ & 537 & 2014 & Asia \\
\hline \multicolumn{5}{|c|}{ Other Parts of World } \\
\hline Richard F O'reilly et al $^{17}$ & $25.6 \%$ & 2442 & 2005 & Australia \\
\hline Jean Marie Januel et al, ${ }^{16}$ & $1.2 \%$ & 23475 & 2012 & Europe \\
\hline
\end{tabular}

In the table 3 data shows different incidences between Western, Asian and Indian population. Incidence varies with geographical region, ethnicity, and genetic make-up of the population. Even in India, there is a marked difference in incidence which may be because of sample size. In view of this difference, we infer that more number of studies with large sample sizes, need to be conducted among the different regions of the world, to know the incidence in that particular population, which may further help in prevention and management of DVT and also optimal utilization of resources. We in our institute do follow customized chemoprophylaxis protocol, to meet the needs and requirements of our patients, with a resultant low incidence of DVT and PE.

Postoperative rehabilitation protocol for TKA patients in our Institute also contributes to low incidence. Our dedicated physiotherapy team starts ankle and calf exercises immediately postoperatively. Patients were started quadriceps and hamstrings exercises, knee and ankle ROM exercises, within 24 hours, followed by weight bearing exercises with walker support within 48 hours.

\section{Conclusion}

We in our Institute do follow Customized chemoprophylaxis protocol to meet the needs and requirement of our patients. The chemoprophylaxis protocol was developed by our institution which caters the patient needs; keeping in the mind of their limitation (large rural background, poor socioeconomic status, minimal primary health care at the village) is easy to follow. The low incidence of DVT and PE in our study may be due to patient from active rural background, ethnicity and geographical consideration. In view of variable results of incidence of DVT and PE 
from different regions of the world, more number of studies with large sample size need to be conducted to understand incidence in that particular population. This will help in optimal utilization of resources for better prevention and management of DVT.

\section{Conflict of Interest: None}

\section{References}

1. V Jain, B K Dhaon, A Jaiswal, V Nigam, J Singla. Deep vein thrombosis after total hip and knee arthroplasty in Indian patients. Postgrad Med J. 2004;80:729-731.

2. Prevention of venous thrombosis and pulmonary embolism. NIH Consensus Development. JAMA. 1986;256:744-749.

3. Seung Yeol Lee, Du Hyun Ro, Chin Youb Chung, Kyoung Min Lee, Soon-Sun Kwon, Ki Hyuk Sung, Moon, Seok Park. Incidence of DVT after major lower limb orthpaedic surgery: analysis of nationwide chain Registry. Yonsei Med J. 2015;56(1):139-145.

4. Nachiketan K Dore, Gopi M, Sathish Devadoss, A Devadoss. Incidence of post-operative deep vein thrombosis in patients undergoing joint replacement surgeries of lower limb. International Journal of Orthopaedics Sciences. 2017;3(3):140-144.

5. Dhanesh R. Kamerkar, M. Joseph John, Sanjay C. Desai, Liesel C. Dsilva, and Sadhna J. Joglekar. Arrive: A retrospective registry of Indian patients with venous thromboembolism. Indian J Crit Care Med. 2016;20(3):150-158.

6. Chandra Prakash Pal, Pulkesh Singh, Sanjay Chaturvedi, Kaushal Kumar Pruthi, and Ashok Vij. Epidemiology of knee osteoarthritis in India and related factors. Indian J Orthop. 2016;50(5):518-522.

7. Syam Sundar Junapudi, B. Babu Rao. A comparative study of cardiovascular disease risk factors among urban and rural population South Indian city. Int J Community Med Public Health. 2017;4(12):4623-4625.

8. Elena Losina, Alexander M. Weinstein, William M. Reichmann, Sara A. Burbine, Daniel H. Solomon, Meghan E. Daigle, et al. Lifetime risk and age of diagnosis of symptomatic knee osteoarthritis in the US. Arthritis Care Res (Hoboken). 2013;65(5):10.1002/acr.21898.

9. Jawahir A Pachore, Shrinand V Vaidya, Chandrasekhar J Thakkar, Haresh Kumar P Bhalodia, and Hemant M Wakankar. ISHKS joint registry: A preliminary report. Indian J Orthop. 2013;47(5):505-509.

10. V Bagaria, N Modi, A Panghate, S Vaidya. Incidence and risk factors for development of venous thromboembolism in Indian patients undergoing major orthopaedic surgery: results of a prospective study. Postgrad Med J. 2006;82:136-139.

11. Piovella F, Wang CJ, Lu H, Lee K, Lee LH, Lee WC, Turpie AG, Gallus AS, Planès A, Passera $\mathrm{R}$, Rouillon A. Deep-vein thrombosis rates after major orthopedic surgery in Asia. An epidemiological study based on postoperative screening with centrally adjudicated bilateral venography. $J$ Thromb Haemost. 2005;3(12):2664-70.

12. Ye Sun, Dongyang Chen, Zhihong Xu, Dongquan Shi, Jin Dai, Jianghui Qin, Qing Jiang. Incidence of Symptomatic and Asymptomatic Venous Thromboembolism after Elective Knee Arthroscopic Surgery: A Retrospective Study with Routinely Applied Venography. Arthroscopy. 2014;30(7):818-22.

13. D. J. Quinlan, J. W. Eikelboom, O. E. Dahl, B. I. Eriksson, P. S. Sidhu and J. Hirsh. Association between asymptomatic deep vein thrombosis detected by venography and symptomatic venous thromboembolism in patients undergoing elective hip or knee surgery. Journal of Thrombosis and Haemostasis. 2007;5:14381443.

14. Agarwala S, Bhagwat AS, Modhe J. Deep vein thrombosis in Indian patients undergoing major lower limb surgery. Indian J Surg. 2003;65:159-62.

15. Won, Y.S., Kim, M., Jun, K.W., Nam, W.S., Ahn, S., Hwang, J.-K., Kim, S.-D., Park, S.-C., Yun, S.S., Lee, W.-C., Park, J.-S. and Kim, J.Y. (2014) Incidence and Clinical Characteristics of Deep Vein Thrombosis (DVT) after Total Knee Arthroplasty (TKA) with DVT Chemoprophylaxis. World Journal of Cardiovascular Diseases. 2014;4:531-538.

16. Jean-Marie Januel, RN, Guanmin Chen, Christiane Ruffieux, Hude Quan, James D. Douketis, FRCPC Mark A. Crowther, FRCPC Cyrille Colin, William A. Ghali, Bernard Burnand. MPH for the IMECCHI Group. Symptomatic In-Hospital Deep Vein Thrombosis and Pulmonary Embolism Following Hip and Knee Arthroplasty Among Patients Receiving Recommended Prophylaxis. JAMA. 2012;307:3.

17. Richard F O'Reilly, Ian A Burgess and Bernard Zicat. The prevalence of venous thromboembolism after hip and knee replacement surgery. MJA. 2005;182:154-159.

18. Tom Jose, Murukan Babu, Georgy J Kuruvilla. Incidence of symptomatic Venous Thromboembolism (VTE), with or without chemoprophylaxis, in patients undergoing Total Knee Replacement: A comparative study. JMSCR. 2017;5(7).

How to cite this article: Avulapati S K, Mahalingam S K, Gudaru J, Gudaru K. The incidence of symptomatic deep vein thrombosis and pulmonary embolism in the Indian population, following TKA patients, with postoperative prophylaxis. Indian J Orthop Surg. 2018;4(4):348-353. 